\title{
Buckling Analysis and Stability of Compressed Low-Carbon Steel Rods in the Elastoplastic Region of Materials
}

\author{
Gaioz Partskhaladze (iD, ${ }^{1}$ Ingusha Mshvenieradze ${ }^{D},{ }^{2}$ Elguja Medzmariashvili, ${ }^{2}$ \\ Gocha Chavleshvili $\left(\mathbb{D},{ }^{1}\right.$ Victor Yepes $\mathbb{D D}^{3}{ }^{3}$ and Julian Alcala ${ }^{3}$ \\ ${ }^{1}$ Engineering and Construction Department, Batumi Shota Rustaveli State University, Batumi 6010, Georgia \\ ${ }^{2}$ Department of Civil and Industrial Engineering, Georgian Technical University, Tbilisi 6000, Georgia \\ ${ }^{3}$ Institute of Concrete Science and Technology (ICITECH), Universitat Politècnica de València, Valencia 46022, Spain
}

Correspondence should be addressed to Victor Yepes; vyepesp@cst.upv.es

Received 4 March 2019; Accepted 14 May 2019; Published 29 May 2019

Academic Editor: Abdul Aziz bin Abdul Samad

Copyright (c) 2019 Gaioz Partskhaladze et al. This is an open access article distributed under the Creative Commons Attribution License, which permits unrestricted use, distribution, and reproduction in any medium, provided the original work is properly cited.

\begin{abstract}
This paper presents new approaches for solving a problem of the stability of compressed rods in the elastoplastic working region of materials. It is known that the columns of buildings, supports of engineering devices, drill rods of oil, and gas extraction industry may be subjected to significant risk of stability loss. Nowadays, there are design methods based on test results defining the relations (e.g., critical stresses-slenderness) to avoid this risk due to stability loss, but the precision and limits of definition are not always known. The main objectives of the study were to develop new approaches that would allow specifying the values of critical stresses of compressed elements beyond the proportional limit. The problem of stability of the compressed elements in the elastoplastic region was studied according to the stability theory. The authors suggested an original approach to the issue; in particular, the determination of values of the critical stresses and the finding of the points of the bifurcation were carried out by the tangent established by experimental results and by the approximation of the so-called double modulus. Comparative analysis showed the advantage of the proposed approach, particularly that the new critical curves were located below the curves of Engesser-Karman and Shanley and above the critical curves established by building codes. A new approach for the determination of critical stresses in the elastoplastic region was developed through which the structural reliability and economic efficiency was increased by almost $12 \%$ compared to the existing approaches.
\end{abstract}

\section{Introduction}

For developing modern structural, civil, mechanical, and other fields of engineering, it is necessary to work out very precise calculating methods for the stability of rod systems. Researchers still have a great interest in the failure of structural elements due to the loss of stability.

A bulk-forming process from metallic materials, such as stamping, forging, extrusion, and spinning, is one of the main processes in industrial manufacturing. Some forging tools, such as supports, punches, or ejectors, may be subjected to significant risk of stability loss (buckling) when they are used. Rods are used for drilling in oil and gas fields. The stability loss of the drill rod is one of the complicating factors of the drilling process, which is a major problem for oil and gas extraction industry [1]. In the seismic areas, steel frames are known as an efficient arrangement for structures. In addition, load-carrying capacity of the columns as the main parts of the frame systems, using limit state design, is determined by the stability in the elastoplastic region of the material [2]. Therefore, steel columns have been widely used to dissipate seismic energy in seismic regions. These considerations are especially relevant in calculating the stability of high-rise buildings taking into account the complicated technological load on them [3-8].

This paper presents new approaches for solving buckling problems for axially compressed columns in the elastoplastic working region of the material. A prismatic (rectangular) steel column was studied as a sample that is depicted in Figure 1. The study of the buckling behavior of prismatic 


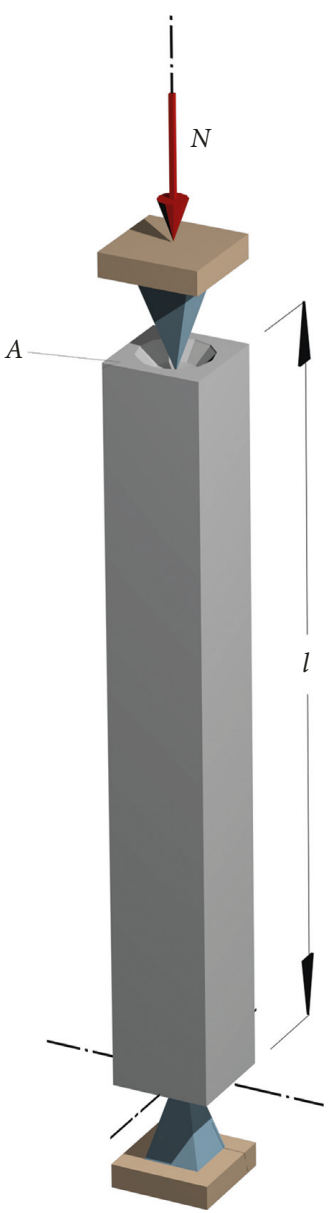

FIgURE 1: A prismatic (rectangular) pin-ended steel column.

(rectangular) compressed elements is a relevant step towards understanding and assessing the reliability of more complex structures [9].

In steel columns, the issues of buckling can be divided into three types: overall buckling, local buckling, and interactive buckling $[10,11]$. In prismatic columns with rectangular cross section and medium slenderness ratio, overall buckling is normally observed first [12].

The buckling appearance (loss of stability) process was assessed with critical forces (from which the values of reduction factor are defined). The reduction factor approach defined from critical stresses appears in two alternative methods in the standards: (a) traditional method using semiempirical design equations and (b) computer-aided method based on linear buckling analysis [12, 13].

It is common knowledge that the physical action causing the buckling of axially compressed columns is the depletion of its lateral stiffness to a value of zero. There are a number of problems in actual engineering situations, which complicate the prediction of buckling loads, e.g., initial (geometric) imperfection, material nonlinearity, and loading eccentricity. The linear elastic Euler model of a prismatic simply supported rod is still the fundamental tool for studying buckling stability $[9,10]$.

In a similar way, several empirical models, for instance, Engesser's [7, 13, 14], are proposed in the literature to experimentally characterize the elastic-plastic and plastic buckling. Later, Shanley $[9,15]$ offered a different approach for defining the buckling process with its semiempirical design equation.

In the literature $[16,17]$, researchers presented a numerical method for computing the load-deflection relationships of loaded square columns with various geometric parameters, loading eccentricity, and column slenderness ratios.

As mentioned above, steel frames are an efficient arrangement for structural systems in seismic regions. For this reason, they have been the subject of strong research interest in recent years. In practice, earthquakes generate multidirectional ground motions, with the occurrence of yielding and buckling effects $[4,6,8]$. Previous research studies $[3,18]$ indicate how the development of local buckling restricts the ability of the members to deform plastically; also how cross-section geometry influences strength, ductility, and the energy absorption process.

In literature [12], the researchers examined the simply supported steel member with uniform cross section subjected to uniform forces. The results showed buckling resistance for such members and were standardized by the reduction factor based on the Ayrton-Perry formula [19] and calibrated by strong theoretical and empirical background. The local and overall stability of the columns through simulating their geometric and material nonlinear behavior was investigated [11]. A methodology [20] was devised to estimate the elastoplastic properties of metallic materials from the hardness measurements using the two most frequently used sharp indenters, i.e., the Vickers indenter and the Knoop indenter. In the study [21], the researchers studied overall buckling behavior and design of high-strength steel columns. The study results indicated that with an increase of the yield strength of high-strength steel, compressive residual stresses ratio to the yield strength becomes evidently lower and possesses much less severe effects on the overall buckling behavior. Different $\mathrm{H}$-shaped sections of the welded steel columns were experimentally and numerically investigated with their overall buckling behavior, and their practical application was promoted [22].

The main disadvantage of the previously proposed design methods to avoid risk due to loss of stability in the elastoplastic region was that the accuracy and limits of the definition were not always known.

The main objectives of the study were to (a) develop the approaches that would allow specifying the values of critical stresses of compressed elements beyond their proportional limit, (b) approximate and specify maximally the theoretical and real values of critical loads, (c) find the possible points of bifurcation in the elastoplastic region, and (d) eliminate the existence of residual deformations in the cross-section and eventually reduce the mass of the structure and increase its reliability.

The problem of the stability of the compressed elements was studied according to the stability theory. Methods based on the equations of equilibrium of mechanics for slightly perturbed systems around the initial position were used to study the stability. 
During the study, these steps were followed:

(a) The energy method was used

(b) The principle of initial imperfections of the rod was considered

(c) Euler's analytical method was used in (during) compression, which, in turn, was based on Hooke's law

(d) The process of stability loss (buckling) was studied depending on the critical stresses and the slenderness ratio $[12,23-26]$

The advantage of the proposed approach in determining stability of the compressed elements compared to the existing ones is that the critical stresses in the elastoplastic region are derived analytically and they accurately reflect the experimental data.

\section{Stability Problem Formulation}

2.1. General Aspects of Understanding Stability. The loadcarrying capacity of a compressed rod could be depleted as a result of stability loss or as a result of the deflection that occurs before the rod fails due to compression. The equilibrium of a rigid body could be stable, unstable, or neutral. The same condition occurs in deformable body mechanics. The only difference is that the type of equilibrium depends on the value of the acting load $[8,27]$.

Figure 2 depicts a long thin (slender) cantilevered rod with axial force loading. The rod is compressed by a relatively small value of the force $N$ and is present in a stable equilibrium since it assumes a slight deviation from the vertical as a result of any perturbed impact, and after the removal of the force, it soon returns to the initial state (Figure 2(a)).

The rod gradually returns to the initial position together with the increase of the load, and the state of the neutral equilibrium occurs at a certain value of the critical force $N_{\mathrm{cr}}$. The rod obtains equilibrium in the deflected position during the modest deviation from the vertical position (dashed line, Figure 2(b)). At this time, the bifurcation (the coexistence of contiguous equilibrium forms) is formed, which is characterized by an "exchange" of stability between two equilibrium forms of the rod.

The rectilinear form "loses" stability, and the curvilinear form still "tries" to obtain it. As it is known, the force that transfers the rod from the rectilinear stable position to the curvilinear stable position is a critical force $N_{\mathrm{cr}}$.

The new equilibrium curvilinear form theoretically becomes stable at loading, the value of which exceeds critical one (Figure 2(c)). This condition is practically unacceptable since the rod already works not for pure compression but for compression with bending, i.e., when the force reaches its critical value, the rectilinear form of the rod is already unstable. The rod will bend in a less rigid plane and obtain (get) a stable curvilinear form. The curvature of the rod begins to increase rapidly even when the load increases slightly, and it loses its load-carrying capacity $[2,13]$.

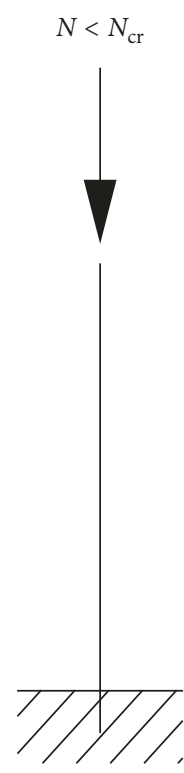

(a)

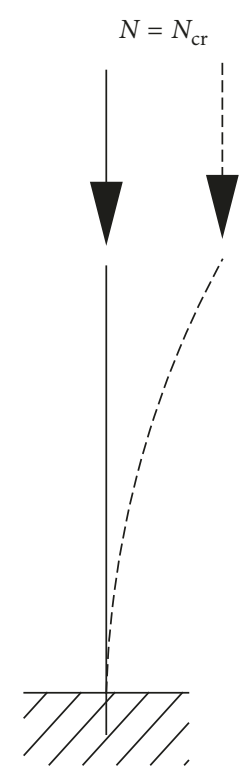

(b)

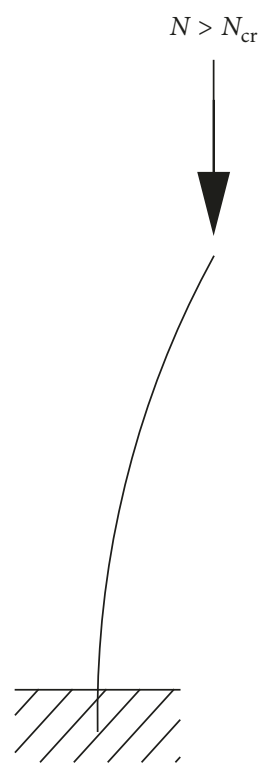

(c)
Figure 2: Equilibrium types of deformed body-cantilevered column under axial force. (a) Stable equilibrium; (b) neutral equilibrium; (c) unstable equilibrium.

2.2. Theoretical Analysis and Euler's Critical Curves in the Elastic Region. Figure 3(a) depicts the long narrow pinended rod on which the compressive force is loaded along the symmetrical axis. Taking into account the principle of initial imperfections of the rod, the rod is bent during the load by the axial force (Figure 3(b)), [12, 24]. In order to reveal the internal bending moment $M$, it is necessary to cut the column in the middle zone for creating the free body (Figure 3(c)).

Moment equilibrium dictates that the bending moment is given by $M=-N y$. Here, $y$ is the lateral deflection. From the resistance of materials, it is known that $M / E I \approx d^{2} y / d x^{2}$. The above two relationships provide defining differential equation for Euler buckling. In addition, according to the energy method, the compressive force reaches its critical value when the ends of the rod approach each other, and the work performed by external forces is equal to the deformation work of the bending of the compressed rod $[6,13,14]$.

A differential equation of the curved axis for such rods is shown as follows:

$$
\frac{d^{2} y}{d x^{2}}+\frac{N}{E I} y=0
$$

By solving it, the formula for calculating critical force/ stress was developed by Leonard Euler [9, 12]:

$$
\begin{gathered}
N_{\mathrm{cr}}=\frac{\pi^{2} E I_{\min }}{l_{0}^{2}}, \\
\sigma_{\mathrm{cr}}=\frac{\pi^{2} E}{\lambda^{2}}
\end{gathered}
$$

where $N_{\text {cr }}$ is the critical force, $\sigma_{\text {cr }}$ is the critical stress, $E$ is the modulus of elasticity (Young's modulus), $\lambda=l_{0} / i_{\min }$ is the 


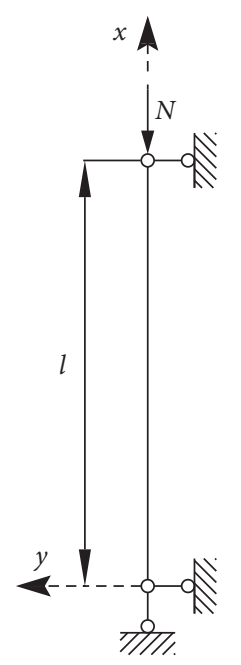

(a)

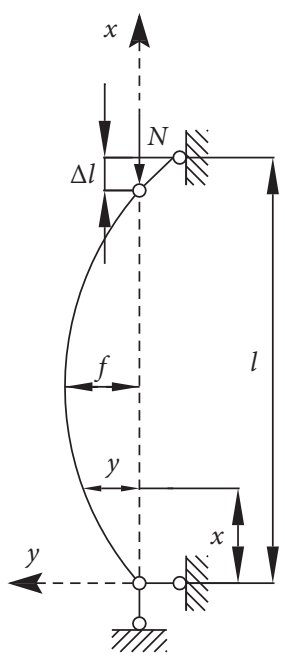

(b)

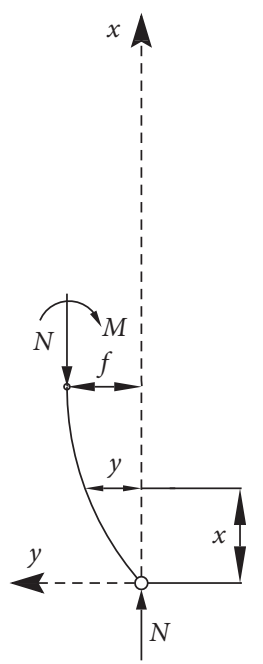

(c)
FIGURE 3: The long slender pin-ended rod/column. (a) Design scheme of a compressed pinned rod; (b) buckling of a long slender rod; (c) free body for Euler buckling model.

slenderness ratio that is equal to the ratio of the effective length of the column to the least of radius gyration, $l_{0}=\mu l$ is the effective length of the $\mathrm{rod} /$ column, $\mu$ is the reduction ratio, which depends on the boundary conditions at the ends of the rod/column, the radius of gyration is equal to $i_{\min }=\sqrt{I_{\min } / A}, I_{\min }$ is the minimum moment of inertia (the second moment of area) of the cross section, and $A$ is the area of the cross section of the column $\sigma_{0}=N / A$.

The geometrical and material characteristics of the carbon steel (S235) columns with various grades are presented in Table 1. A diagram depicting the stress-strain relationship for carbon steel is constructed in Figure 4(a) according to Table 1, and a diagram depicting the slenderness ratio of critical stresses (equation (2)) for carbon steel is constructed in Figure 4(b), Curve $1[9,15,21,28]$.

Equation (2) is valid for such rods that work in the elastic region. Critical stresses $\left(\sigma_{\mathrm{cr}}\right)$ are less than the proportional limit $\left(\sigma_{\mathrm{pl}}\right), \sigma_{\mathrm{cr}}<\sigma_{\mathrm{pl}}$ in this region (Figure $4(\mathrm{a})$ ), and the slenderness ratio $(\lambda)$ is higher than the critical slenderness ratio $\left(\lambda_{\mathrm{cr}}\right), \lambda>\lambda_{\mathrm{cr}}$ for carbon steel. For example, the diagram constructed on Figure 4(b) depicts that the elastic region corresponds to $\lambda \in[102, \ldots, 200]$, the zone of slenderness for steel-S235. Here, $\lambda_{\mathrm{cr}}=\pi \sqrt{E / \sigma_{\mathrm{pl}}}$, where $\sigma_{\mathrm{pl}}$ is the proportional limit of the material. The constant modulus of elasticity (Young's modulus) $E$ spreads in the whole cross section of the rod. A diagram constructed in the zone of the large slenderness is called Euler's quadratic hyperbola (Figure 4(b), Curve 1) [9]. The points of bifurcation are located at the points existing on the hyperbola diagram. It shows the coexistence of the neighboring equilibrium forms for long flexible rods (i.e., columns with slender proportions) working in the elastic region.

2.3. Critical Curves and Theoretical Analysis in the Elastoplastic Region. When a perfectly straight column is
TABLE 1: Design parameters of carbon steel with various grades.

\begin{tabular}{lccccc}
\hline$\varepsilon \times 10^{8}$ & $\sigma\left(\mathrm{kN} / \mathrm{cm}^{2}\right)$ & $\begin{array}{c}E_{\mathrm{t}} \times 10^{-4} \\
\left(\mathrm{kN} / \mathrm{cm}^{2}\right)\end{array}$ & $T \times 10^{-4}\left(\mathrm{kN} / \mathrm{cm}^{2}\right)$ & $\lambda_{\mathrm{T}}$ & $\lambda_{\mathrm{t}}$ \\
\hline 0.95 & 20 & 2.06 & 2.06 & 102 & 102 \\
1.0 & 21 & 1.42 & 1.72 & 90 & 81.8 \\
1.1 & 22 & 0.99 & 1.39 & 79 & 66.6 \\
1.2 & 22.8 & 0.67 & 1.05 & 67.6 & 54 \\
1.3 & 23.4 & 0.46 & 0.85 & 59 & 44 \\
1.4 & 23.8 & 0.26 & 0.54 & 47.5 & 32.7 \\
1.5 & 23.9 & 0.13 & 0.33 & 37 & 23.1 \\
1.6 & 24 & 0.06 & 0.19 & 28 & 15.7 \\
$1.8-4.0$ & 24 & 0 & 0 & 0 & 0 \\
4.5 & 24.1 & 0.02 & 0.07 & 17 & 9.1 \\
5 & 24.2 & 0.04 & 0.13 & 23 & 12.7 \\
6 & 24.7 & 0.05 & 0.15 & 24.5 & 14.2 \\
8 & 25.75 & 0.05 & 0.15 & 24 & 13.8 \\
10 & 26.85 & 0.05 & 0.15 & 23.6 & 13.6 \\
12 & 28 & 0.05 & 0.15 & 23 & 13.5 \\
\hline
\end{tabular}

subjected to an axial force, the state of internal stresses near the midpoint of the rod/column is uniform compression. For a crooked column (the principle of initial imperfections), the state of internal stress near the midpoint is not uniform, and there arises the net tension stress on the outer curve of the column $[12,24]$.

Figure 5 depicts a blowup of the middle slenderness ratio of the column and the stress distribution in the cross section of the middle zone (Figure 5(a)). The tensile zone, i.e., the unloaded zone (area $A_{1}$ in Figure 5(b)) together with the compressed zone (area $A_{2}$ in Figure 5(b)), arises in the cross section during buckling in which (tensile zone) constant modulus of elasticity $E$ will exist because of the relatively small value of tensile stresses and Gerstner's law (see the zone, where $\sigma<\sigma_{\text {el }}$, Figure 4(a)) $[9,27]$.

There is no increase in compressive force during the buckling in the elastoplastic region in accordance with the first theory of calculating the stability of compressed elements. The compressive stresses will transfer from the unloaded zone to the compressed zone due to the crookedness of the column $[18,29]$. Consequently, the stresses will be increased in the compressed zone, and instead of the constant modulus of elasticity $E$, the smaller value of the tangent modulus $E_{\mathrm{t}}$ will exist (Figure 5(b)). Thus, in the cross section, there will be simultaneously two elastic moduli. The modulus $E$ will exist in the tensile zone, and the tangent modulus $E_{\mathrm{t}}$ will exist in the compressed zone (Figure 5(b)).

Only the constant modulus of elasticity $E$ is no longer useful in the elastoplastic working region of the material, that is, the zone of slenderness $-\lambda \in[40, \ldots, 102]$ for the steel S235 in the cross section, because the stress-strain relationship in this region becomes nonlinear (Figures 4(a) and 5(b) compressed zone) [22, 30].

The formula for calculating critical stresses for the elastoplastic region is identical to the calculation formula for the elastic region. There is just one difference between them, e.g., instead of the constant elastic modulus $E$, the reduced modulus $T$ of Engesser-Karman appears, which has the following form in the case of a rectangular cross section $[13,27]$. 


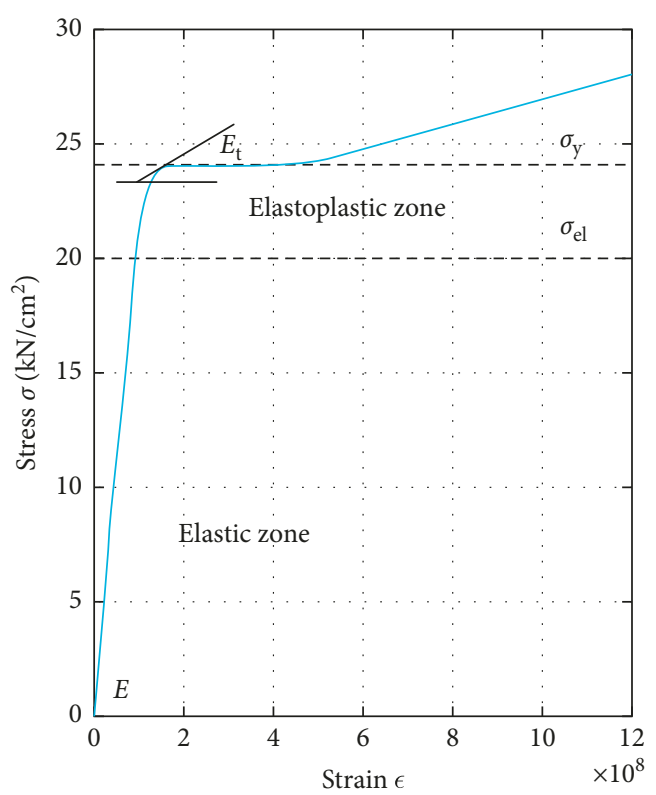

(a)

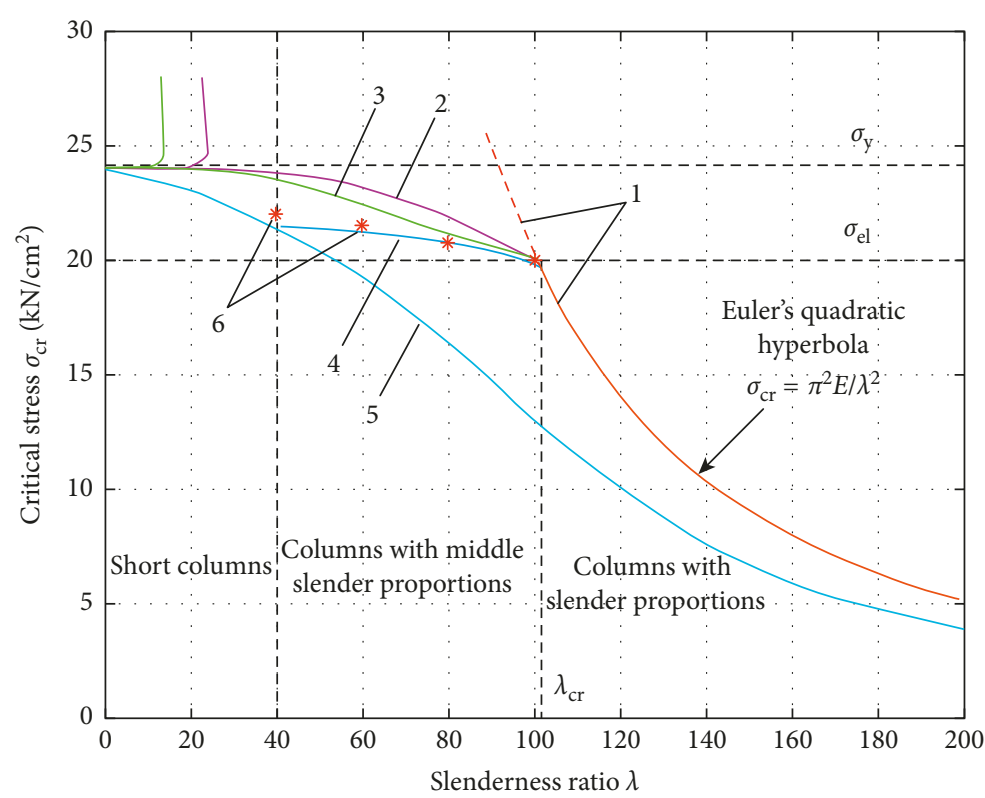

(b)

Figure 4: Blown up middle zone of a long slender rod. (a) Free body for Euler buckling model; (b) combined axial and bending stresses in a crooked column.

$$
T=\frac{4 E E_{\mathrm{t}}}{\left(\sqrt{E}+\sqrt{E_{\mathrm{t}}}\right)^{2}} .
$$

In this case, when using equation (3), Euler's formula will be equal to the following:

$$
\sigma_{\mathrm{cr}}=\frac{\pi^{2} T}{\lambda^{2}} .
$$

Curve 2 in Figure 4(b) depicts the dependency diagram $\left[\sigma_{\mathrm{cr}}-\lambda\right]$ in the zone of medium slenderness $\lambda \in[40, \ldots, 102]$ constructed by the use of equation (4).

If the sum of the moments of the internal forces will be equal to the external moment, then the formula of the bending moment for any cross section, with allowance for the so-called tangent modulus, will be equal to the following:

$$
\begin{aligned}
M= & \int_{A_{1}} \sigma_{1} y d A+\int_{A_{2}} \sigma_{2} y d A \Leftrightarrow M=\int_{A_{1}} \frac{E}{\rho} y^{2} d A \\
& +\int_{A_{2}} \frac{E_{\mathrm{t}}}{\rho} y^{2} d A \Leftrightarrow M=\frac{E}{\rho} \int_{A_{1}} y^{2} d A+\frac{E_{\mathrm{t}}}{\rho} \int_{A_{2}} y^{2} d A .
\end{aligned}
$$

Hence,

$$
M=\frac{1}{\rho}\left(E I_{1}+E_{\mathrm{t}} I_{2}\right),
$$

where $I_{1}$ and $I_{2}$ are the moments of inertia of both parts of the cross section towards the neutral axis (Figure 6). The tangent modulus $E_{\mathrm{t}}$ is obtained as constant (an average).
As commonly known, the formula will be obtained using the elastoplastic modulus $T^{*}$ instead of the elastic modulus $E$ in the formula $M=E I / \rho$ of the bending moment.

$$
M=\frac{T^{*} I}{\rho},
$$

where $M$ is the bending moment, $T^{*}$ is the double modulus, $I$ is the moment of inertia, and $\rho$ is the local bending radius (the radius of bending at the current section).

The formula for the double modulus of elasticity is derived by equating equations (6) and (7) and by solving $T^{*}$ [7] as follows:

$$
T^{*}=\frac{1}{I}\left(E I_{1}+E_{\mathrm{t}} I_{2}\right) .
$$

As is obvious from equation (8), the double modulus depends on the shape of the cross section of the rod. The neutral axis divides the cross section into parts having various moduli that allow finding the reduced stiffness $\left(T^{*} \cdot I\right)$ of the rod as the product of the moment of inertia $(I)$ of the rod on the reduced modulus $\left(T^{*}\right)$.

According to the second approach for calculating the stability of compressed elements (Shanley's theory), there will not be the unloaded zone in the cross section in the elastoplastic region of the material since the compressive force increases along with the bending [13].

At the beginning of stability loss, the loading speed with the longitudinal forces of the rod will be much more than the unloading speed of the bent fibers and the stresses will increase throughout the section, i.e., a small bend will not lead to unloading. Therefore, above the limits of elasticity, the critical tangential force will be the tangent according to modulus $E_{\mathrm{t}}$ 


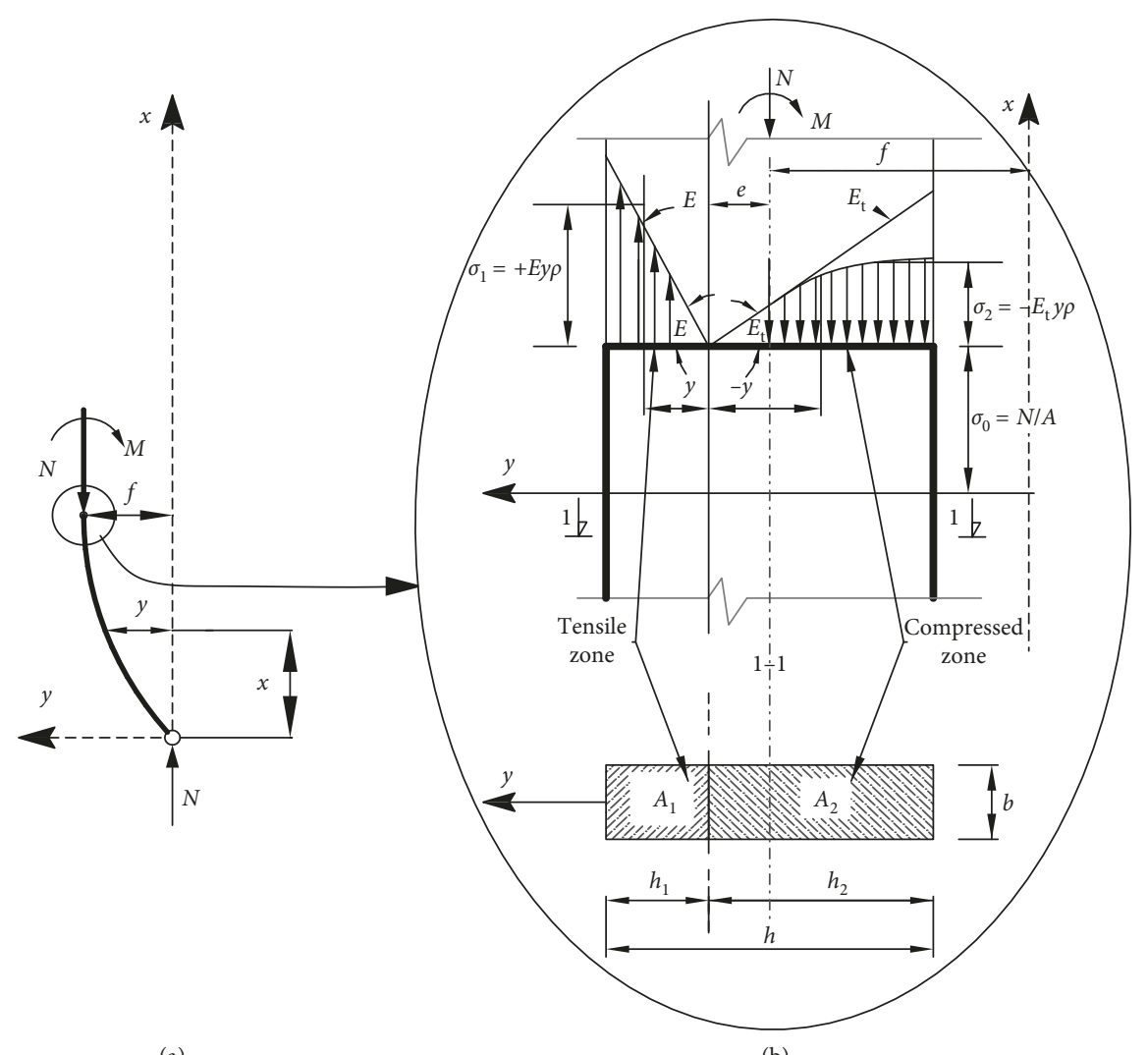

(a)

(b)

Figure 5: Euler diagram with a real elastoplastic behavior using a specific variation of stresses for carbon S235 steel. (a) Stress-strain diagram; (b) dependence of the critical stresses and the slenderness ratio. (1) Euler's quadratic hyperbola; (2) Engesser-Karman's model; (3) Shanley's model; (4) authors approach; (5) model of building codes; (6) experimental data.

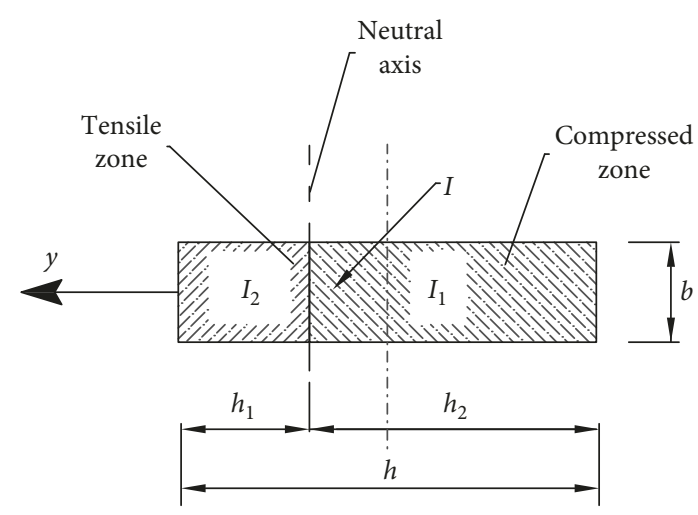

FIGURE 6: The cross section of the rod.

[7]. This assumption greatly simplifies the calculation of the compressed rods on critical forces, especially the difficult tasks of the stability of slabs and shells [15].

Then, the parabolic equation proposed by Shanley and proven by Stelmakh's tests can be used for the tangent modulus of various steels at the elastoplastic stage.

$$
E_{\mathrm{t}}=E\left[1-\left(\frac{\sigma_{\mathrm{crt}}-\sigma_{\mathrm{el}}}{\sigma_{\mathrm{y}}-\sigma_{\mathrm{el}}}\right)^{2}\right]
$$

where $\sigma_{\text {crt }}$ is the critical stress in the elastoplastic region, $\sigma_{\mathrm{el}}$ is the elastic limit, and $\sigma_{\mathrm{y}}$ is the yield strength.
On the contrary, the critical stress calculated by Engesser can be expressed with the dependence of the slenderness, $\lambda$, and the tangent modulus, $E_{\mathrm{t}}$. In this case, Euler's formula will be as follows [14, 27]:

$$
\sigma_{\mathrm{cr}}=\frac{\pi^{2} E_{\mathrm{t}}}{\lambda^{2}}
$$

Curve 3 in Figure 4(b) depicts the dependency graph $\left[\sigma_{\mathrm{cr}}-\lambda\right]$ in the zone of medium slenderness $\lambda \in[40, \ldots, 102]$ constructed by equation (10) and according to Table 1.

\section{Problem Solution}

3.1. Choice of Design Loads and Criteria for Stability. In addition to the methods presented in the introduction, when studying the rod deformations, small deviations compared to the length of the rod are adopted, or when studying rod deformation, it is assumed that the rod is in a geometrically linear form. The stress-strain relation is known. Despite all this, the problem includes many difficulties. The first difficulty is to determine the points of bifurcation. The second difficulty is to determine which of the loads, the tangent modulus $\left(E_{\mathrm{t}}\right)$, or the reduced modulus (Engesser-Karman$T$ ) must be chosen for the calculation. The third difficulty is how to eliminate residual deformations in the cross section. It is necessary to obtain such a dependence, which by a single approach will determine such critical stresses, which will be 
close to the real values of critical loads. They will also be close to the experimental data.

In order for the critical curves to be determined not only in the elastoplastic region on the basis of the experimental data, the authors proposed a new approach to the analytical solution of the formula for calculating critical stresses.

\subsection{Proposed New Approach for Obtaining Critical Stresses in} the Elastoplastic Region and Its Theoretical Analysis. The calculation methods based on the results of the experimental research have been the most common up to now. Therefore, various authors have proposed formulas for calculating the longitudinal bending beyond the limits of elasticity [12].

The authors suggested an original approach to the issue; in particular, the determination of values of the critical stresses and the finding of the points of the bifurcation were carried out by the tangent $\left(E_{t}\right)$ established by experimental results and by the approximation of the so-called double modulus $\left(T^{*}\right)$.

If the modulus of elasticity $E$ is replaced by the double modulus $T^{*}$ in the Euler's formula, the critical stress will have the following form:

$$
\sigma_{\mathrm{cr}}=\frac{\pi^{2} T^{*}}{\lambda^{2}}
$$

In this study, the authors substituted the value of the double modulus $\left(T^{*}\right)$ from equation (8) into the formula of the critical stress (equation (11)), and it was assumed that Euler's critical stress is valid until the critical slenderness $\left(\lambda_{\mathrm{cr}}\right)$.

The cross section was divided into the tensile and compressed zones. The constant modulus of elasticity $E$ will be used for the tensile zone and the tangent modulus $E_{t}$ from equation (9) established by Shanley and proven by Stelmakh's tests in the compressed zone.

The joint work of both moduli in the cross section (modulus of elasticity $E$ in the tensile zone and the tangent modulus $E_{\mathrm{t}}$ in the compressed zone) was used in the double modulus $\left(T^{*}\right)$ (equation (8)) to obtain the following equation:

$$
\sigma_{\mathrm{cr}}=\frac{\pi^{2} T^{*}}{\lambda^{2}}=\frac{\left(\pi^{2} \cdot 1\right) / I\left(E I_{1}+E_{\mathrm{t}} I_{2}\right)}{\lambda^{2}}=\frac{\pi^{2} E}{\lambda_{\mathrm{cr}}^{2}} \frac{I_{1}}{I}+\frac{\pi^{2} E_{\mathrm{t}}}{\lambda_{t}^{2}} \frac{I_{2}}{I} .
$$

From equation (12), the following equation is obtained:

$$
\sigma_{\mathrm{cr}}=\sigma_{\mathrm{cr}}^{\prime} \frac{I_{1}}{I}+\sigma_{\mathrm{crt}} \frac{I_{2}}{I}
$$

where $\sigma_{\text {cr }}^{\prime}$ is the critical stress when the critical slenderness within the elasticity reaches the limit value $\left(\lambda_{\mathrm{cr}}=102\right)$, and $\lambda_{\mathrm{t}} \leq \lambda_{\mathrm{cr}}$ is the critical slenderness that has low value in comparison with $\lambda_{\text {cr }}$ (Figure 4(b)).

$\sigma_{\text {crt }}$ is the critical stress for the tangent modulus (in the elastoplastic region) and is equal to the following:

$$
\sigma_{\mathrm{crt}}=\frac{\pi^{2} E_{\mathrm{t}}}{\lambda_{\mathrm{t}}^{2}}
$$

Input the value of $E_{\mathrm{t}}$ from (9) into equation (14). In this case, the formula of $\sigma_{\text {crt }}$ will be as follows:

$$
\sigma_{\mathrm{crt}}=\frac{\pi^{2} E}{\lambda_{\mathrm{t}}^{2}}\left[1-\left(\frac{\sigma_{\mathrm{crt}}-\sigma_{\mathrm{el}}}{\sigma_{\mathrm{y}}-\sigma_{\mathrm{el}}}\right)^{2}\right] \text {. }
$$

The following quadratic equation is obtained by solving $\sigma_{\text {crt }}$ from equation (15):

$$
\sigma_{\mathrm{crt}}^{2}-\left[2 \sigma_{\mathrm{el}}-\frac{\left(\sigma_{\mathrm{y}}-\sigma_{\mathrm{el}}\right)^{2}}{\pi^{2} E} \lambda_{\mathrm{t}}^{2}\right] \sigma_{\mathrm{crt}}+\sigma_{\mathrm{y}}\left(2 \sigma_{\mathrm{el}}-\sigma_{\mathrm{y}}\right)=0 .
$$

The value of the critical stress- $\sigma_{\text {crt }}$ was solved from quadratic equation (16) and substituted in equation (13), and thus, the adjusted formula for calculating the critical stresses was obtained in the elastoplastic working region of the material.

$$
\begin{aligned}
\sigma_{\mathrm{cr}}= & \frac{\pi^{2} E}{\lambda^{2}}\left(1-\frac{I_{2}}{I}\right)+\left(\sigma_{\mathrm{el}}-\frac{\lambda_{\mathrm{t}}^{2}\left(\sigma_{\mathrm{y}}-\sigma_{\mathrm{el}}\right)^{2}}{2 \pi^{2} E}\right) \frac{I_{2}}{I} \\
& +\frac{I_{2}}{I} \sqrt{\left(\sigma_{\mathrm{el}}-\frac{\lambda_{\mathrm{t}}^{2}\left(\sigma_{\mathrm{y}}-\sigma_{\mathrm{el}}\right)^{2}}{2 \pi^{2} E}\right)-\sigma_{\mathrm{y}}\left(2 \sigma_{\mathrm{el}}-\sigma_{\mathrm{y}}\right) .}
\end{aligned}
$$

3.3. Numerical Analysis. According to equation (17) of the critical stresses obtained by the authors, the dependence between the slenderness and the critical stresses in the region of medium slenderness is constructed as Curve 4 in Figure 4(b).

The following data were used for obtaining the numerical values:

The material: carbon steel (S235); proportional limit $\sigma_{\mathrm{pl}}=19.6 \mathrm{kN} / \mathrm{cm}^{2}$; elastic limit $\sigma_{\mathrm{el}}=20 \mathrm{kN} / \mathrm{cm}^{2}$; yielding limit $\sigma_{\mathrm{y}}=24 \mathrm{kN} / \mathrm{cm}^{2}$; modulus of elasticity $E=21000 \mathrm{kN} / \mathrm{cm}^{2}$; design resistance $R_{\mathrm{y}}=23.5 \mathrm{kN} / \mathrm{cm}^{2}$; slenderness ratio $40 \leq$ $\lambda_{\mathrm{t}} \leq 102$; critical slenderness ratio $\lambda_{\mathrm{cr}} \approx 102$.

At the elastoplastic region and middle slenderness ratio, $\lambda \in[40, \ldots, 102]$; from Figure 4(b), it is seen that the curve of critical stresses constructed according to the reduced modulus $(T)$ of Engesser-Karman equation (4) is above all the graphs.

Curve 3 of the critical stresses (equation (10)) determined by the tangent modulus $\left(E_{\mathrm{t}}\right)$ of Shanley is located below Curve 2 (Figure 4(b)).

Curve 4 of the critical stresses (equation (17)) obtained by approximating the double modulus proposed by authors is located below the curve of Shanley, which is very close to the results obtained by the experimental data (Figure 4(b), asterisks-6). It confirms the accuracy of the new approach (Figure 4(b)).

Curve 5 depicts values of critical stresses defined by building codes for the rectangular cross section (Figure 4(b)).

On the basis of theoretical and numerical analysis, the following results were obtained: 
(1) A new approach to the determination of critical stresses in the elastoplastic region was developed, as a result of which the values of critical stresses with a high accuracy were determined.

(2) The locations of possible points of the bifurcationbuckling in the elastoplastic region were established. These bifurcation points are on Curve 4 (authors approach) because this curve is the closest to the real bifurcation points, i.e., the experimental data.

(3) The approach for the elimination of the presence of residual deformations in the cross section was developed because Curve 4 in Figure $4(\mathrm{~b})$ is below Curve 2 and Curve 3 and closer to the experimental data.

(4) Approximation and accuracy of theoretical and real critical loads were arisen because Curve 4 is the closest to the real critical loads, i.e., the experimental data.

(5) The results obtained from the proposed new approaches indicate that the structure reliability was enhanced by almost $10-12 \%$ in comparison with existing approaches.

(6) Economic efficiency was increased by almost $12-14 \%$ in comparison with the building codes. Since the new critical curve is located above the curves determined by building codes, which is defined by using a safety factor gained empirically.

(7) The study of the buckling behavior of prismatic (rectangular) compressed elements is a relevant step towards understanding and assessing the reliability of more complex structures. For the column of other cross sections, e.g., circular and open-section, the formula for calculating critical stress (equation (17)) remains the same, only some geometrical characteristics need to be changed, e.g., moments of inertia.

\section{Conclusions}

From the results of this study, the following conclusions can be made:

(1) It is recommended to use the tangent modulus- $E_{t}$ (equation (9)) compared with the reduced modulus$T$ (equation (3)) in determining the critical stresses in the elastoplastic region

(2) It is recommended to use the new approach proposed by the authors to determine the stability of the compressed rod in the elastoplastic region, where the values of the critical stresses (equation (17)) are determined by approximation of the double modulus $T^{*}$ (equation (8)) and the tangent modulus $E_{\mathrm{t}}$ (equation (9)) established by the results of experimental studies

(3) A new approach to the determination of critical stresses, proposed by the authors, increases reliability by almost $10-12 \%$
(4) In rods (columns) of medium slenderness, the points below the critical curve obtained as a result of new approaches will not experience stability loss and the development of residual plastic deformations, and the bifurcation points will be located on this Curve 4 (Figure 4(b))

(5) A new approach to the determination of critical stresses, proposed by the authors, achieves economic efficiency by almost $12-14 \%$

(6) Reduction of the mass of the structure, respectively, reduces $\mathrm{CO}_{2}$ emissions

\section{Data Availability}

The data used to support the findings of this study are available from the corresponding author upon request.

\section{Conflicts of Interest}

The authors declare that there are no conflicts of interest regarding the publication of this paper.

\section{Acknowledgments}

This research was financially supported by the Erasmus Mundus Action 2 Project "Electra: Enhancing Learning in ENPI Countries through Clean Technologies and Research related Activities" (project: ELEC1400294) and the Spanish Ministry of Economy and Competitiveness, along with FEDER funding (project: BIA2017-85098-R).

\section{References}

[1] D. J. Braun, "On the optimal shape of compressed rotating rod with shear and extensibility," International Journal of NonLinear Mechanics, vol. 43, no. 2, pp. 131-139, 2008.

[2] B. Rossi and K. J. R. Rasmussen, "Carrying capacity of stainless steel columns in the low slenderness range," Journal of Structural Engineering, vol. 139, no. 6, pp. 1088-1092, 2013.

[3] X. Cheng, Y. Chen, L. Niu, and D. A. Nethercot, "Experimental study on $\mathrm{H}$-section steel beam-columns under cyclic biaxial bending considering the effect of local buckling," Engineering Structures, vol. 174, pp. 826-839, 2018.

[4] Y. Goto, M. Muraki, and M. Obata, "Ultimate state of thinwalled circular steel columns under bidirectional seismic accelerations," Journal of Structural Engineering, vol. 135, no. 12, pp. 1481-1490, 2009.

[5] M. J. Leamy, "Wave based analysis of buckling in columns and frames," in Proceeding of the 22nd Reliability, Stress Analysis, and Failure Prevention Conference; 25th Conference on Mechanical Vibration and Noise, International Design Engineering Technical Conferences and Computers and Information in Engineering Conference, vol. 8, ASME, Portland, OR, USA, 2013.

[6] J. Lu, B. Wu, and Y. Mei, "Buckling mechanism of steel core and global stability design method for fixed-end bucklingrestrained braces," Engineering Structures, vol. 174, pp. 447461, 2018.

[7] A. G. Razdolsky, "Revision of Engesser's approach to the problem of Euler stability for built-up columns with batten plates," Journal of Engineering Mechanics, vol. 140, no. 3, pp. 566-574, 2014. 
[8] D. G. Zapata-Medina, L. G. Arboleda-Monsalve, and J. D. Aristizabal-Ochoa, "Static stability formulas of a weakened Timoshenko column: effects of shear deformations," Journal of Engineering Mechanics, vol. 136, no. 12, pp. 1528-1536, 2010.

[9] A. Ziółkowski and S. Imiełowski, "Buckling and post-buckling behaviour of prismatic aluminium columns submitted to a series of compressive loads," Experimental Mechanics, vol. 51, no. 8, pp. 1335-1345, 2010.

[10] P. Li, X. Liu, and C. Zhang, "Interactive buckling of cablestiffened steel columns with pin-connected crossarms," Journal of Constructional Steel Research, vol. 146, pp. 97-108, 2018.

[11] L. Yang, G. Shi, M. Zhao, and W. Zhou, "Research on interactive buckling behavior of welded steel box-section columns," Thin-Walled Structures, vol. 115, pp. 34-47, 2017.

[12] F. Papp, "Buckling assessment of steel members through overall imperfection method," Engineering Structures, vol. 106, pp. 124-136, 2016.

[13] P. D. Simão, "Influence of shear deformations on the buckling of columns using the generalized beam theory and energy principles," European Journal of Mechanics-A/Solids, vol. 61, pp. 216-234, 2017.

[14] X.-F. Li and K. Y. Lee, "Effects of Engesser's and Haringx's hypotheses on buckling of Timoshenko and higher-order shear-deformable columns," Journal of Engineering $\mathrm{Me}$ chanics, vol. 144, no. 1, article 04017150, 2018.

[15] J. Becque, "Inelastic plate buckling," Journal of Engineering Mechanics, vol. 136, no. 9, pp. 1123-1130, 2010

[16] M. Ahmed, Q. Q. Liang, V. I. Patel, and M. N. S. Hadi, "Nonlinear analysis of rectangular concrete-filled double steel tubular short columns incorporating local buckling," Engineering Structures, vol. 175, pp. 13-26, 2018.

[17] Y.-L. Long and L. Zeng, "A refined model for local buckling of rectangular CFST columns with binding bars," Thin-Walled Structures, vol. 132, pp. 431-441, 2018.

[18] C. D. Moen, A. Schudlich, and A. von der Heyden, "Experiments on cold-formed steel C-section joists with unstiffened web holes," Journal of Structural Engineering, vol. 139 , no. 5, pp. 695-704, 2013.

[19] J. Szalai, "Complete generalization of the Ayrton-Perry formula for beam-column buckling problems," Engineering Structures, vol. 153, pp. 205-223, 2017.

[20] C. Zhang, F. Li, and B. Wang, "Estimation of the elasto-plastic properties of metallic materials from micro-hardness measurements," Journal of Materials Science, vol. 48, no. 12, pp. 4446-4451, 2013.

[21] H. Ban and G. Shi, "Overall buckling behaviour and design of high-strength steel welded section columns," Journal of Constructional Steel Research, vol. 143, pp. 180-195, 2018.

[22] T.-Y. Ma, Y.-F. Hu, X. Liu, G.-Q. Li, and K.-F. Chung, "Experimental investigation into high strength Q690 steel welded $\mathrm{H}$-sections under combined compression and bending," Journal of Constructional Steel Research, vol. 138, pp. 449-462, 2017.

[23] A. Kervalishvili and I. Talvik, "Modified procedure for buckling of steel columns at elevated temperatures," Journal of Constructional Steel Research, vol. 127, pp. 108-119, 2016.

[24] T. Tankova, J. P. Martins, L. Simões da Silva, L. Marques, H. D. Craveiro, and A. Santiago, "Experimental lateraltorsional buckling behaviour of web tapered I-section steel beams," Engineering Structures, vol. 168, pp. 355-370, 2018.

[25] N. Tullini, A. Tralli, and D. Baraldi, "Buckling of Timoshenko beams in frictionless contact with an elastic half-plane,"
Journal of Engineering Mechanics, vol. 139, no. 7, pp. 824-831, 2013.

[26] B. Xie, J. Hou, Z. Xu, and M. Dan, "Component-based model of fin plate connections exposed to fire-part I: plate in bearing component," Journal of Constructional Steel Research, vol. 149, pp. 1-13, 2018.

[27] J. D. Aristizabal-Ochoa, "Stability of columns with semi-rigid connections including shear effects using Engesser, Haringx and Euler approaches," Engineering Structures, vol. 33, no. 3, pp. 868-880, 2011.

[28] F. M. Mitenkov, V. G. Bazhenov, V. K. Lomunov, and S. L. Osetrov, "Effects of elasticity, plasticity, and geometrical nonlinearity in problems of static and dynamic bending of plates," Doklady Physics, vol. 56, no. 12, pp. 622-625, 2011.

[29] J. Bielski and B. Bochenek, "On a compressed elastic-plastic column optimized for post-buckling behaviour," Engineering Optimization, vol. 40, no. 12, pp. 1101-1114, 2008.

[30] O. Fergani, I. Lazoglu, A. Mkaddem, M. El Mansori, and S. Y. Liang, "Analytical modeling of residual stress and the induced deflection of a milled thin plate," International Journal of Advanced Manufacturing Technology, vol. 75, no. 1-4, pp. 455-463, 2014. 


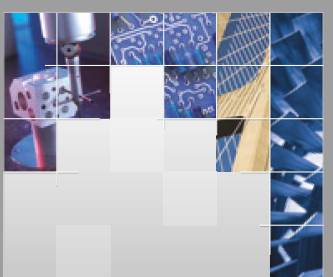

\section{Enfincering}
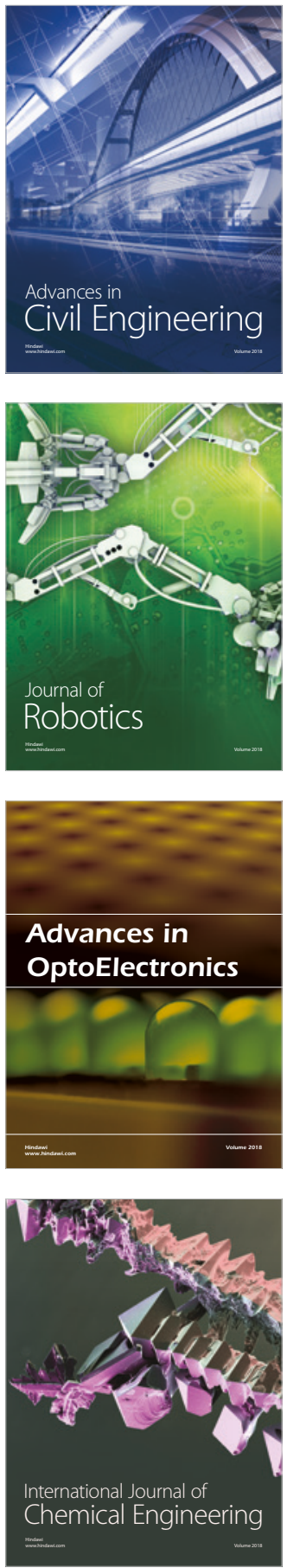

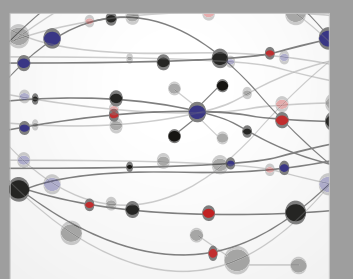

\section{Rotating \\ Machinery}

The Scientific World Journal

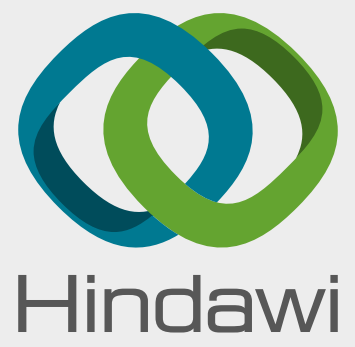

Submit your manuscripts at

www.hindawi.com
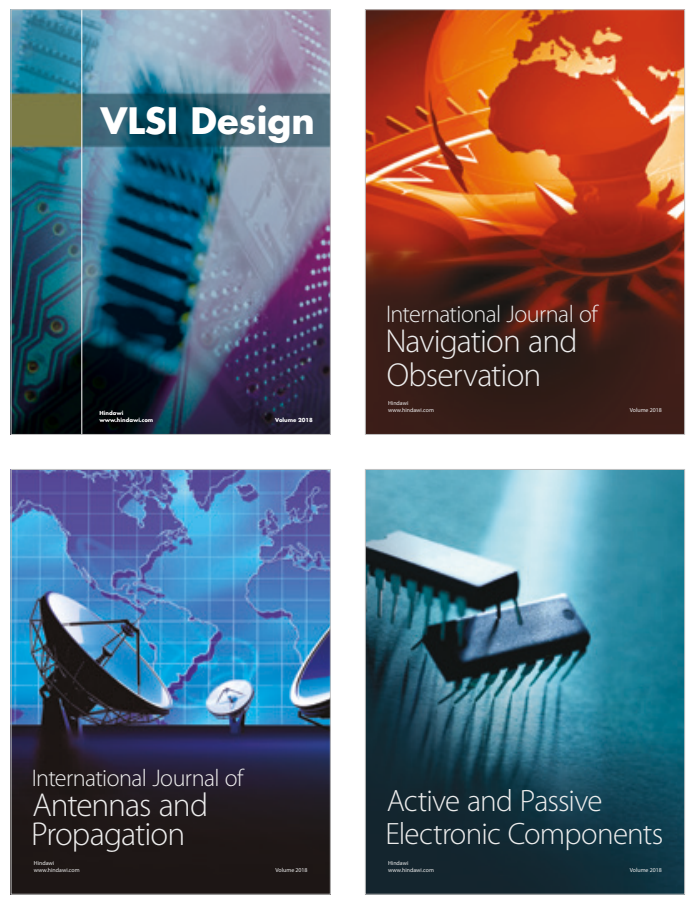
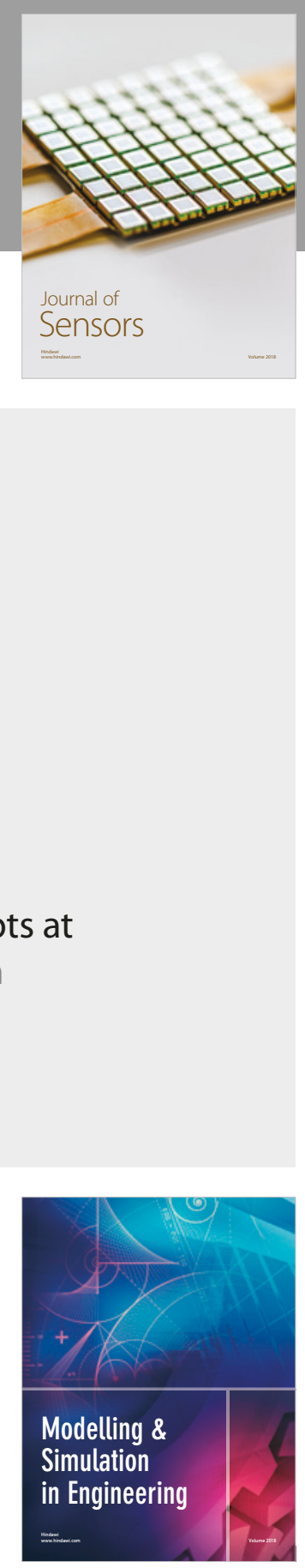

\section{Advances \\ Multimedia}
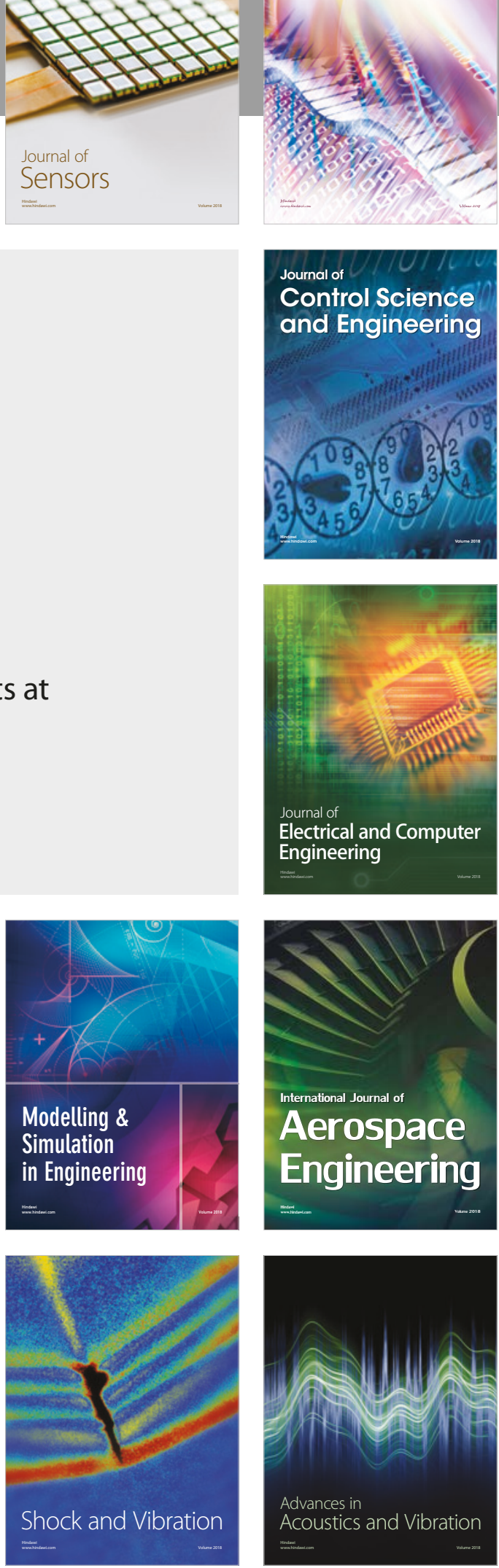